Article

\title{
Electrospraying, a Reproducible Method for Production of Polymeric Microspheres for Biomedical Applications
}

\author{
Nathalie Bock ${ }^{1,2,3, *}$, Maria A. Woodruff ${ }^{1}$, Dietmar W. Hutmacher ${ }^{2}$ and Tim R. Dargaville ${ }^{3}$ \\ 1 Biomaterials and Tissue Morphology Group, Institute of Health and Biomedical Innovation, \\ Queensland University of Technology, Kelvin Grove, 4059, Queensland, Australia; \\ E-Mail: mia.woodruff@qut.edu.au \\ 2 Regenerative Medicine Group, Institute of Health and Biomedical Innovation, Queensland \\ University of Technology, Kelvin Grove, 4059, Queensland, Australia; \\ E-Mail: dietmar.hutmacher@qut.edu.au \\ 3 Tissue Repair and Regeneration Program, Institute of Health and Biomedical Innovation, \\ Queensland University of Technology, Kelvin Grove, 4059, Queensland, Australia; \\ E-Mail: t.dargaville@qut.edu.au \\ * Author to whom correspondence should be addressed; E-Mail: n.bock@qut.edu.au; \\ Tel.: +61-7-313-86165; Fax: +61-7-313-86100.
}

Received: 11 November 2010; in revised form: 3 December 2010 / Accepted: 21 December 2010 / Published: 5 January 2011

Abstract: The ability to reproducibly load bioactive molecules into polymeric microspheres is a challenge. Traditional microsphere fabrication methods typically provide inhomogeneous release profiles and suffer from lack of batch to batch reproducibility, hindering their potential to up-scale and their translation to the clinic. This deficit in homogeneity is in part attributed to broad size distributions and variability in the morphology of particles. It is thus desirable to control morphology and size of non-loaded particles in the first instance, in preparation for obtaining desired release profiles of loaded particles in the later stage. This is achieved by identifying the key parameters involved in particle production and understanding how adapting these parameters affects the final characteristics of particles. In this study, electrospraying was presented as a promising technique for generating reproducible particles made of polycaprolactone, a biodegradable, FDA-approved polymer. Narrow size distributions were obtained by the control of electrospraying flow rate and polymer concentration, with average particle sizes ranging from 10 to $20 \mu \mathrm{m}$. Particles were shown to be spherical with a homogeneous embossed 
texture, determined by the polymer entanglement regime taking place during electrospraying. No toxic residue was detected by this process based on preliminary cell work using DNA quantification assays, validating this method as suitable for further loading of bioactive components.

Keywords: electrospraying; microspheres; polycaprolactone; drug delivery

\section{Introduction}

The use of polymeric particles has been of great interest in the biomedical field for the last 50 years, with a particular niche present in the field of drug delivery [1,2]. Current technologies allow the production of biodegradable nano- and micro-sized materials able to encapsulate therapeutic molecules to be gradually released through diffusion and degradation in vivo. The motivation behind this process is the possibility to overcome the limitations faced in bolus delivery of molecules, especially proteins where the harsh in vivo environment can cause denaturation, shortening their half-life after delivery, and thus reducing action efficacy [3]. Polymeric particles are therefore presented as reservoir systems able to protect the proteins from their environment, enhancing their long-term biological activity. Ideally, these systems are also able to provide tailored release rates, required by certain therapies, by the control of particle morphology, size and polymeric matrix [4]. Importantly, such particles have the potential to minimize the propagation of drug payloads to non-targeted areas, limiting unwanted effects and allowing a site-specific delivery [1-3,5].

Several techniques have been presented in the literature for fabrication of polymeric nano- and microparticles, the most popular being based on emulsion techniques [5-10]. Molecules are dispersed or dissolved into a polymer solution and emulsified to form micro-droplets that are further dried after solvent removal [11]. However, the use of organic solvents, unless carefully controlled, is a drawback in many of these techniques since it can lead to denaturation of protein-based drugs during processing, increasing the variability in encapsulation efficiencies and loading capacities [6]. Secondly, the size distributions of particles fabricated by emulsion-based techniques tend to be inhomogeneous and broad, contributing to their lack of reproducibility, which in turn hinders their clinical use. Size distribution is a crucial parameter and it was shown that monodisperse size would enable a better control of release profiles and bioavailability of the loaded drug in the body [12,13]. Particle morphology is also important since it affects the internalization by non-phagocytic cells and the degradation of the polymer matrix which, in turn, determines the release kinetics of the loaded component. Therefore, homogeneity of morphology is also an important consideration to ensure particles with reproducible characteristics are obtained.

Fabrication of polymer microparticles by electrospraying has the potential to overcome the limitations of emulsion-based techniques and to provide reproducibly loaded nano- and microparticles [14]. Electrospraying is a one-step technique which has potential to generate narrow size distributions of submicrometric particles, with limited agglomeration of particles and high yields [15]. The principles of electrospraying are based on the ability of an electric field to deform the interface of a liquid drop, 
established by Lord Rayleigh in 1882 [16], further developed by Zeleny in 1917 [17] and Sir Taylor in 1964 [18]. The theory of charged droplets states that if an electrified field is applied to any droplet, the electric charge generates an electrostatic force inside the droplet, known as the Coulomb force, which competes with the cohesive force intrinsic to the droplet. When the applied Coulomb force is able to overcome the cohesive force of the droplet manifested in the surface tension, the droplet will undergo breakup into smaller droplets in the micro- to nano-scales. This phenomenon begins at the Taylor Cone, referring to the progressive shrinkage of the unstable, charged macro-droplet into a cone from which the smaller charged droplets will be ejected as soon as the surface tension is overcome by the Coulomb force. Rayleigh predicted a limit to determine the breakup of the droplet, called the Rayleigh limit, $\mathrm{L}_{\mathrm{R}}$, expressed in Equation (1). $\mathrm{L}_{\mathrm{R}}$ is a function of $q$ the surface charge of the droplet, $\varepsilon$ the permittivity of the surrounding medium, $\gamma$ the surface tension of the liquid and $R$ the radius of the droplet. The maximum surface charge of the droplet is given by Equation (2). Based on these equations, monodisperse electrosprayed particles can be fabricated by using the appropriate parameters.

$$
\begin{aligned}
& L_{R}=q\left(64 \pi^{2} \varepsilon \gamma R^{3}\right) \\
& q=8 \pi \sqrt{\varepsilon_{0} \gamma R^{3}}
\end{aligned}
$$

Electrospinning is based on the same principles of charged droplets involving the ejection of a nano-jet instead of droplets from the Taylor Cone. The difference between the electrospinning and electrospraying techniques lies in the chain entanglement density of the polymer solution [19]. Previous studies have demonstrated that a critical polymer concentration called $c_{o v}$ can dictate the behavior of electrospraying/electrospinning [20]. This critical concentration can be found for each type of polymer solution and represents the critical chain overlap concentration where entanglement begins to occur. In order to produce fibers, the polymer concentration, $c$, must be chosen such that a threshold ratio $c / c_{o v}$ is overcome. Therefore, at low chain entanglement density, electrospraying of droplets instead of electrospinning of fibers will occur at the Taylor Cone. The $c / c_{o v}$ ratio must be determined experimentally for each type of polymer, for example $c / c_{o v}$ for poly(methylmethacrylate) is between 3 and 10 depending on the molecular weight distribution of the polymer chains in solution [20].

The electrospraying process is conceptually simple: a polymer solution is loaded into a syringe and infused at a constant rate using a syringe pump through a small but highly charged capillary (e.g., a 16-26 gauge needle). The applied voltage used is typically up to + or $-30 \mathrm{kV}$ and the collector might be placed at a 7 to $30 \mathrm{~cm}$ distance from the capillary. Once the droplets have detached from the Taylor cone, the solvent evaporates, generating dense and solid particles, propelled towards the collector. In the context of drug loading, the bioactive molecule is mixed to the polymer solution before electrospraying and can further be emulsified [21]. Some studies which have been undertaken include encapsulation of hydrophilic and hydrophobic model drugs [12], model proteins [21-25], antibiotics [14,26] and anti-cancer drugs [13] in polylactide (PLA) [12,21], poly(lactic-co-glycolic acid) (PLGA) [24,26], polycaprolactone (PCL) [13,24] and chitosan [14].

During the electrospraying process, there are several parameters which all have an inter-dependent influence on viscosity, electrical conductivity, particle size, distribution, encapsulation efficiencies, loading capacities and in vitro release profiles [12,14,15,21-22,24,27]. These parameters include voltage, distance to collector, needle gauge, flow rate, polymer, drug, solvent, surfactant, 
protein/polymer ratio and organic/aqueous ratio. As a consequence, although electrospraying is a promising technique, the number of parameters to be used can render its optimization highly complex. The characteristics of electrosprayed particles are still not completely understood and it is important to proceed in a step-wise manner intended to understand the relationship between processing parameters and characteristics of electrosprayed microparticles before one progresses to the inclusion of highly fragile and expensive bioactive molecules. This study details the reproducibility of the process and identifies the key parameters responsible for particle size, distribution and morphology as a prelude to using the validated methodologies for the loading of a bioactive molecule.

Previous studies have correlated the effects of key variables of electrospraying [26-30]. Most of these studies are PLGA-based, which are well-known as the most common biodegradable and FDA-approved polymers in tissue engineering literature. However, in the context of drug delivery for orthopedic applications, a slower degrading polymer like polycaprolactone (PCL) should be considered. PCL is also FDA-approved and various drugs have been encapsulated in PCL microspheres and nanospheres [31,32]. PCL is highly permeable to small drug molecules and degrades through its ester linkages. As compared to PLGA-based polymers, it also presents the advantage of a lesser acidic environment being generated during degradation [31], however, only very few studies have investigated the production of electrosprayed PCL particles [33-35]. As aforementioned, the type of polymer will give different characteristics of electrosprayed particles and due to the complexity and inter-dependence of variables involved in the process, PLGA production parameters might not be translatable to PCL. The objective of this study was therefore to study the morphology and particle size obtained for non-loaded electrosprayed PCL microspheres and to ensure their reproducibility. Importantly, the toxicity of so-produced microspheres was assessed in order to ensure that no toxic residue remained after electrospraying. These are essential steps to be validated and understood before progressing to protein loading.

\section{Experimental Section}

\subsection{Materials}

Polycaprolactone (PCL), $\mathrm{Mn}=50,000 \mathrm{kDa}$ (Perstorp Ltd, UK-Capa ${ }^{\circledR}$ 6500C) was used to produce the microspheres. Ultra-pure chloroform (99.0-99.4\%) from Merck, Germany was used to dissolve PCL. Different concentrations were prepared: 5, 7.5, 9 and $10 \% \mathrm{w} / \mathrm{v}$ (i.e., for $10 \% \mathrm{w} / \mathrm{v}, 10 \mathrm{~g}$ of PCL were dissolved in chloroform and made up to $100 \mathrm{~mL}$ total volume). The polymer solutions were magnetically stirred for 3 hours at room temperature to allow complete dissolution before electrospraying.

\subsection{Microsphere Production}

Figure 1(a) shows a typical schematic of the electrospraying setup used to produce the microspheres. Initially, the electrospraying parameters chosen in this work were based on previous studies on optimization of electrosprayed particles [15,26-30,33,36,37]. Temperature and relative humidity ranged from 22 to $24{ }^{\circ} \mathrm{C}$, and 44 to $49 \%$ respectively. Collectors were made of standard aluminum foils $\left(20 \times 20 \mathrm{~cm}^{2}\right)$. PCL solutions were loaded in a $2.5 \mathrm{~mL}$ glass syringe (Hamilton, USA) 
fitted with a 21 or 26-gauge stainless steel nozzle (Terumo, Japan and Becton Dickinson, USA). PCL solutions were extruded through the nozzle at a constant rate of either 0.2 or $0.5 \mathrm{~mL} / \mathrm{h}$ using a syringe pump (WPI, USA). The tip-to-collector (TTC) distance was set to 15, 20 or $25 \mathrm{~cm}$ respectively. High-voltage was applied between the needle and collector ranging from 10 to $18 \mathrm{kV}$. After electrospraying, the collectors were placed under vacuum for a further 72 hours, to remove any chloroform residue from microspheres. The microspheres were then transferred into glass vials and further evacuated for storage. The different experimental parameters employed are summarized in Table 1. The microspheres produced under each set of parameters are abbreviated as $\mathrm{M}$, with a number referring to the condition type (a set of parameters). For example: for condition 1, the produced microspheres are M1-type.

Figure 1. (a) Schematic of a typical electrospraying setup. (b) Picture of the aluminum foil collected after the electrospraying process. (c) Dried microspheres collected from the aluminum foil and further placed in a glass vial for storage. (d) Microspheres taken from the glass vial and analyzed on a microscope slide. (e) Picture of the electrospraying process, inside the safety box, comprising the syringe pump, syringe loaded with polymer solution, collector and electrodes. (f) Picture of the power supply located outside the safety box.
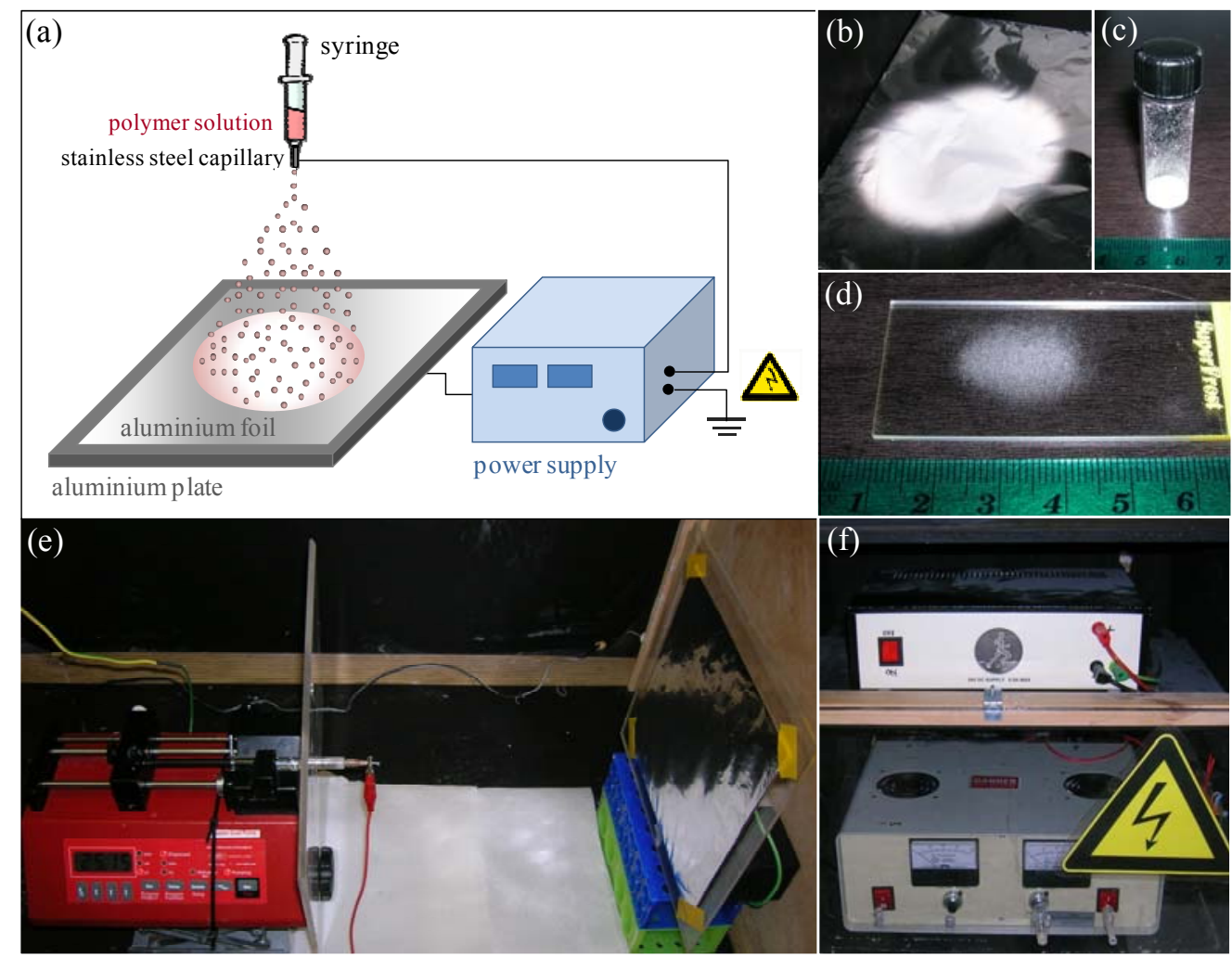
Table 1. Set of parameters tested for each condition.

\begin{tabular}{lccccc} 
& Gauge & PCL Concentration $(\% \mathrm{w} / \mathrm{v})$ & Voltage $(\mathrm{kV})$ & TTC Distance $(\mathrm{cm})$ & Feed Rate $(\mathrm{mL} / \mathrm{h})$ \\
\hline Condition 1 (M1) & 26 & 5 & 10 & 20 & 0.5 \\
Condition 2 (M2) & 26 & 7.5 & 10 & 20 & 0.5 \\
Condition 3 (M3) & 26 & 9 & 10 & 25 & 0.5 \\
Condition 4 (M4) & 26 & 10 & 10 & 15 & 0.5 \\
Condition 5 (M5) & 26 & 10 & 10 & 20 & 0.5 \\
Condition 6 (M6) & 26 & 10 & 10 & 20 & 0.2 \\
Condition 7 (M7) & 26 & 10 & 10 & 25 & 0.5 \\
Condition 8 (M8) & 21 & 10 & 10 & 25 & 0.5 \\
Condition 9 (M9) & 26 & 10 & 10 & 25 & 0.2 \\
Condition 10 (M10) & 26 & 10 & 16 & 25 & 0.5 \\
Condition 11 (M11) & 26 & 10 & 16 & 25 & 0.2 \\
Condition 12 (M12) & 26 & 10 & 18 & 25 & 0.5 \\
Condition 13 (M13) & 26 & 10 & 18 & 25 & 0.2 \\
\hline
\end{tabular}

\subsection{Physical Characterization}

The morphology and microstructure of electrosprayed microspheres were characterized with a scanning electron microscope (FEI Quanta 200 SEM) operating at $10 \mathrm{kV}$. Micrographs were taken from low and high magnifications, in order to have overviews of batches and detailed morphology of microspheres respectively. Microspheres were imaged directly on the microscope slides used for particle size determination, or after collection from the aluminum foil. In the latter case, particles were carefully deposited on carbon sticky tape, previously mounted on aluminum stubs. Both microscope slides and stubs were gold sputtered (BIORAD SC-500 Sputter coater) for $75 \mathrm{~s}$ at $30 \mathrm{~mA}$ before imaging.

In order to determine particle size of electrosprayed microspheres, a microscope glass slide was introduced in the electrospraying box and held in contact with the collector, in the center of the spraying zone for 5 minutes. The slide was then removed and analyzed by light microscopy (AxoVision, Carl Zeiss MicroImaging GmbH, Germany). In order to assess the reproducibility of electrospraying, 3 replicates of each condition were generated. Voltage was turned off between replicates. Particle size was assessed with Image J analysis software (NIH) based on the micrographs. The results were plotted as box plots and expressed in medians, with $\mathrm{n}=100-1,000$ for each replicate, whereas size distribution was shown for all values obtained per condition.

Electrosprayed particles (M3-type) and unprocessed pellets were characterized by differential scanning calorimetry (TA Instruments Q100 DSC) by scanning from $0{ }^{\circ} \mathrm{C}-110{ }^{\circ} \mathrm{C}-0{ }^{\circ} \mathrm{C}$ with a heating and cooling rate of $10{ }^{\circ} \mathrm{C} / \mathrm{min}$. The initial run was followed by a repeat run with the thermal history erased.

\subsection{Biological Effect of Microspheres}

The effect of electrosprayed microspheres on cells was assessed by two methods: the extraction method and direct contact method as per ISO 10993-12. The extraction method, also known as the elution method, required an extract from the material to be tested. The extract was placed on a 
near-confluent monolayer of fibroblast cells and toxicity was evaluated by observing cell numbers using DNA measures. M3-type microspheres were used for this experiment, UV sterilized for 40 minutes immediately before the assay. 0.1 and $1 \% \mathrm{w} / \mathrm{v}$ of microspheres were placed in completed Dulbecco's modified Eagle medium (DMEM) (10\% fetal bovine serum, 1\% penicillin/streptomycin) for 1 and $24 \mathrm{~h}$. The extract solutions were further removed, filtered and seeded on cells. For the direct contact method, 0.01 and $0.1 \% \mathrm{w} / \mathrm{v}$ M3-type microspheres were rinsed in media for 15 minutes and 1 hour, before being placed on the near-confluent monolayer of fibroblast cells, or left non-rinsed but incubated for the same amount of time $\left(37^{\circ} \mathrm{C}, 5 \% \mathrm{CO}_{2}\right)$. In both methods, NIH3T3 cells were cultured for $24 \mathrm{~h}$ before exposure to the test solutions for another $48 \mathrm{~h}$ (initial seeding density: $3 \times 10^{4}$ cells). DNA quantification was determined using CyQUANT ${ }^{\circledR}$ (Invitrogen) $(n=4)$. Results were expressed in normalized averages \pm SE.

\section{Results and Discussion}

\subsection{Physical Characterization}

\subsubsection{Morphology}

Electrospraying resulted in either microspheres or flattened particles, both with textured surfaces. The spherical morphology was obtained only for high polymer concentrations ( 9 and $10 \% \mathrm{w} / \mathrm{v}$ ), while flattened morphology and coalescence between particles was observed for decreased polymer concentrations (5 and $7.5 \% \mathrm{w} / \mathrm{v}$ ) (Figure 2). These results are in accordance with previous studies, where polymer concentration was often shown to be the most critical parameter in the morphology of electrosprayed particles [33].

Figure 2. Influence of polymer concentration on microsphere morphology: (a) M1-type microspheres $(5 \% \mathrm{w} / \mathrm{v})$, (b) M2-type microspheres $(7.5 \% \mathrm{w} / \mathrm{v})$, (c) M3-type microspheres $(9 \% \mathrm{w} / \mathrm{v}),(\mathbf{d}) \mathrm{M} 4$-type microspheres $(10 \% \mathrm{w} / \mathrm{v})$. Scale bar is $20 \mu \mathrm{m}$.

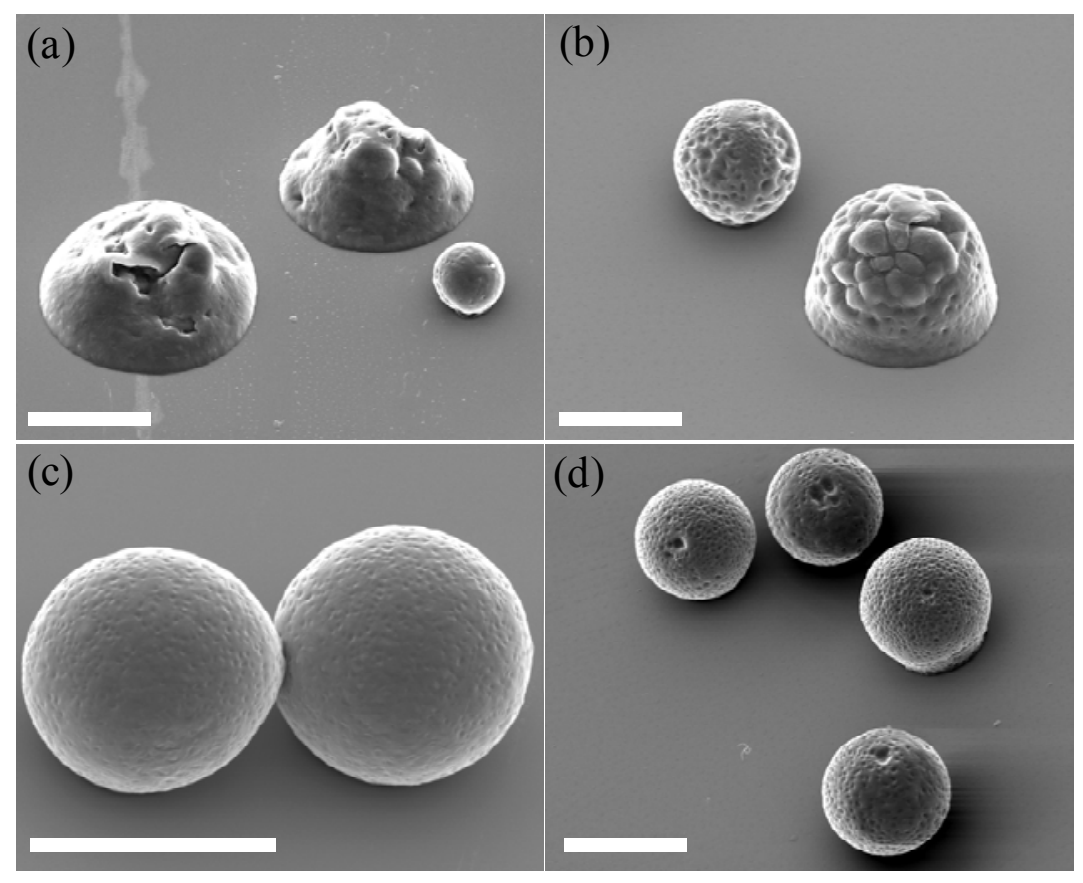


The generation of electrosprayed particles is widely accepted to be controlled by two main mechanisms: solvent evaporation from droplets en route from the tip to the collector, and contemporaneous polymer diffusion during evaporation [28]. Rapid polymer diffusion does not necessarily lead to spherical particles but will ensure solid, dense particles. Both these mechanisms are dictated by the characteristics of the electrosprayed polymer solution itself, dependent on molecular weight and polymer concentration. For conditions 1 and 2 (Table 1) for instance, the polymer solutions are only 5 and $7.5 \% \mathrm{w} / \mathrm{v}$, respectively and lead to the flattened morphology shown in Figure 2(a) and (b), rather than a spherical morphology, observed at higher polymer concentrations ( 9 and $10 \% \mathrm{w} / \mathrm{v})$ as shown in Figure 2(c) and (d). The flattened morphology is even more pronounced for the $5 \% \mathrm{w} / \mathrm{v}$ particles rather than the $7.5 \% \mathrm{w} / \mathrm{v}$ particles, indicating that lower polymer concentration favors the formation of flat particles instead of spheres. These two cases are a direct consequence of incomplete solvent evaporation, since the solvent contents are higher for decreased polymer contents. The electrosprayed particles produced for these concentrations are still partially dissolved when they hit the collector and therefore not fully dried, leading to inhomogeneous semi-solid, flat particles that would further solidify after deposition.

Chain entanglements also are important to the physical properties of the particles produced. At low polymer concentrations, there are less entanglement possibilities for the polymer chains where the operating regime is known as the semidilute unentangled regime. In this state, the concentration is large enough for chains to overlap, but not sufficient to generate a significant degree of entanglement [20]. At higher concentrations, the same available hydrodynamic volume is occupied by more polymer chains, introducing chain entanglements. Gupta et al. defined the crossover of concentration from the semidilute unentangled to semidilute entangled regime as the critical entanglement concentration, $c_{\text {ent }}$, which marks the distinct onset of significant chain entanglements in solution. Therefore in the semidilute unentangled regime: $c_{o v}<c<c_{e n t}$, where $c$ is the polymer concentration and $c_{o v}$ the critical chain overlap concentration $[15,20]$. It is thus essential to use a polymer concentration $>c_{\text {ent }}$ to have the entangled regime.

Reproducibility of electrosprayed particles is a problem when working in the semidilute unentangled regime as the possibility of a distribution of polymer chains in the droplets could generate higher local concentrations in some droplets. This explains why some spherical particles are still shown from low concentration solutions, as seen in Figure 2(a) and (b). In order to obtain reproducible, homogeneous, and solid particles, it is necessary to ensure complete solvent removal and to use polymer concentrations above $c_{\text {ent }}$. This is equally important for producing spherical particles as it was shown that if the evaporating droplets present a sufficiently entangled network before they reach the Rayleigh limit, the resulting particles will remain monodispere and spherical, as the entangled network stabilizes the droplet against rupture, reducing the frequency of smaller offspring particles being emitted [28]. There are some scaling laws to determine $c_{e n t}$ for each type of polymer solution, however it is most likely to be determined experimentally. In the case of PCL $50 \mathrm{kDa}$ dissolved in chloroform, it can be deducted from this study that $c_{e n t}$ is comprised between 7.5 and $10 \% \mathrm{w} / \mathrm{v}$, where $10 \% \mathrm{w} / \mathrm{v}$ was sufficient to produce solid particles on the collector, ensuring homogeneity and sphericity of particles.

The texture observed for spherical particles was previously described as an 'embossing golf-ball structure of the colloidal surface' [35]. Another study on the effect of the solvent properties on electrosprayed polymer particles described how the morphology can be changed according to the type 
of solvent used and its concentration [15]. It was shown that solvents with boiling points $>140{ }^{\circ} \mathrm{C}$, like $\mathrm{N}$,N-dimethylformamide $146{ }^{\circ} \mathrm{C}$, or benzaldehyde $178{ }^{\circ} \mathrm{C}$, would be able to generate smooth surfaces during electrospraying. Chloroform has a much lower boiling point of $61.2{ }^{\circ} \mathrm{C}$, explaining the textured surface that was observed. Such texture was also seen in an even more pronounced way when electrospraying PCL with dichloromethane [33], which boils at $40{ }^{\circ} \mathrm{C}$, corroborating this theory. In the case of in vivo implantation, it is noted that the topographical complexity of a biomaterial is preferred for cell attachment since it generates an increased number of anchoring sites for cells [38]. As a consequence, from this point of view, chloroform can be considered as an adequate solvent to be used in electrospraying of particles to be used in vivo.

Figure 3 illustrates that some of the electrosprayed microspheres presented a certain degree of concomitant fiber formation between the particles. This was shown to occur for the highest polymer concentration $(10 \% \mathrm{w} / \mathrm{v})$ and was favored by lower rates $(0.2$ versus $0.5 \mathrm{~mL} / \mathrm{h})$, indicating that a higher concentration favored the formation of fibers. No differences in fiber formation were seen in the 10 to $18 \mathrm{kV}$ voltage range while feed rate was clearly a determinant in that respect as seen in column 1 $(0.2 \mathrm{~mL} / \mathrm{h})$ compared to column $3(0.5 \mathrm{~mL} / \mathrm{h})$ of Figure 3 . Fibers were extremely thin, on the nanometer scale, acting as a discrete coating on top of the spheres. Such nanofibers were almost non-existent for the $0.5 \mathrm{~mL} / \mathrm{h}$ generated microspheres, while they were more numerous on the $0.2 \mathrm{~mL} / \mathrm{h}$ generated microspheres.

Figure 3. Influence of feed rate and voltage on microsphere morphology with a high and low magnification for each type of microsphere: (a-b) M9-type (c-d) M7-type, (e-f) M11-type, (g-h) M10-type, (i-j) M13-type, (k-l) M12-type. In the first and third columns, scale bar is $10 \mu \mathrm{m}$. In the second and fourth columns, scale bar is $100 \mu \mathrm{m}$.
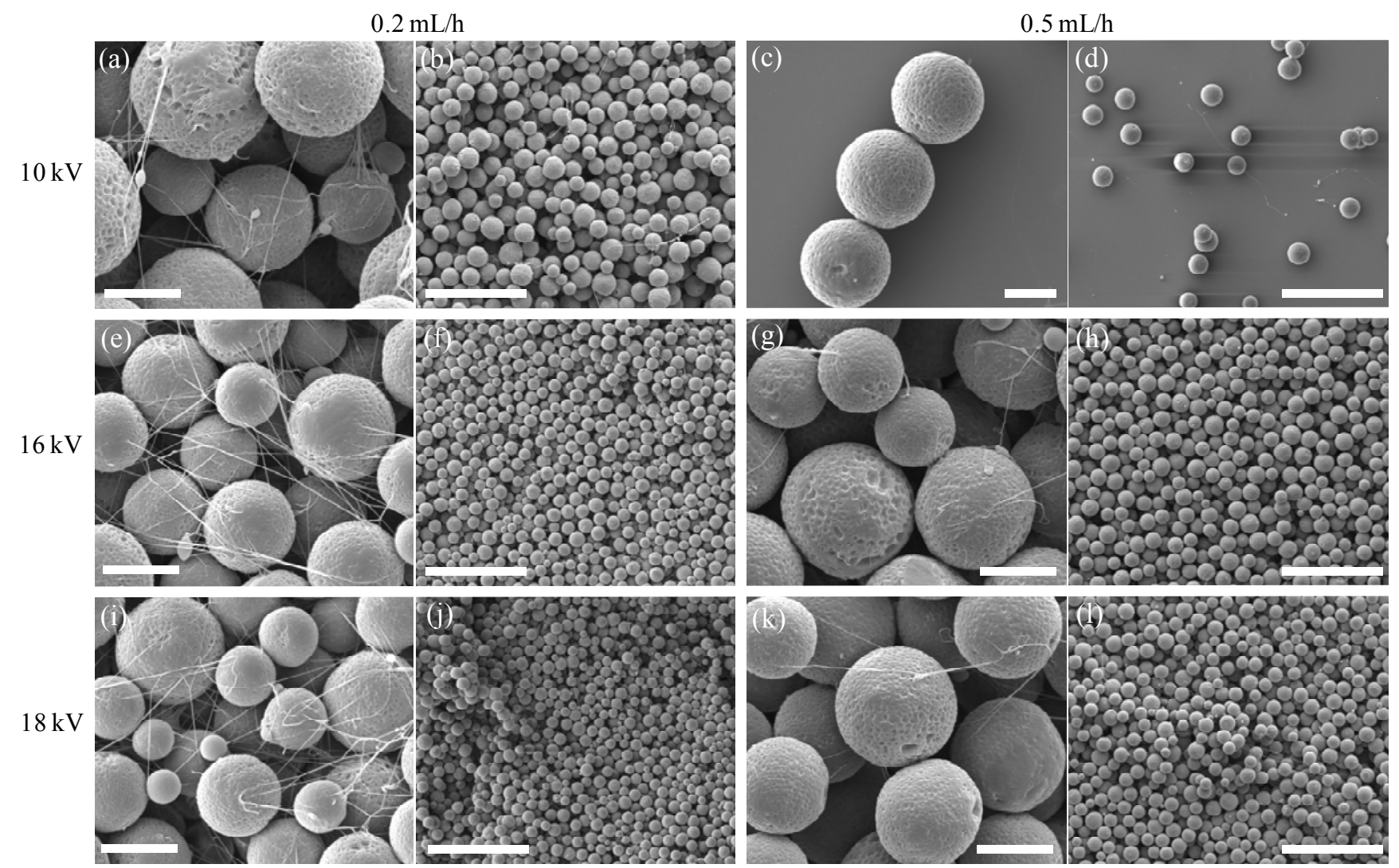
Previously we described the importance of polymer concentration on morphology of electrosprayed particles, since it predicts the semidilute regime that would take place (entangled or unentangled). Here it can be seen that flow rate is also involved in the regime determination. In fact, by transforming the previous concentrations into polymer volume fractions $\varphi$, with $\varphi_{\text {ent }}$ the critical entanglement polymer volume fraction and $\varphi_{\text {ov }}$ the critical chain overlap polymer volume fraction, we can also introduce $\varphi_{\text {Ray }}$, the polymer volume fraction in a droplet at the Rayleigh limit. It was shown by Almería et al. that for $\varphi_{\text {ov }}<\varphi_{\text {Ray }}<\varphi_{\text {ent }}$ in the semidilute unentangled regime, the polymer network can preserve some droplet integrity, but is not strong enough to preserve the particle from deforming via stretching during the fission process. Electrosprayed droplets are likely to undergo Coulomb fission during evaporation, because they shrink without losing charge, and thus are broken up into small, highly charged droplets, called offspring droplets [28]. When $\varphi_{\text {Ray }}>\varphi_{\text {ent, }}$, the droplets are stabilized from such rupture, however when $\varphi_{\text {ov }}<\varphi_{\text {Ray }}<\varphi_{\text {ent, }}$ offspring droplets are ejected, extruding with them a thin charged fiber from the primary droplet, which will remain frozen in this state after evaporation. According to the results, it is likely to be the case for the $0.2 \mathrm{~mL} / \mathrm{h}$ electrosprayed particles (columns $1-2$ of Figure 3 ), inferring that $\varphi_{\text {Ray }}$ is decreasing with decreasing flow rate.

In Equation (3) where $Q$ is the liquid flow rate $\left(\mathrm{m}^{3} \cdot \mathrm{s}^{-1}\right), I$ the current, $\varepsilon_{\text {air }}$ the permittivity of air, $\gamma$ the surface tension of solution in ambient air, and $d$ the initial droplet diameter, $I / Q$ and $d$ can be determined as a function of $Q$ and the polymer solution properties [28]. According to our results we can infer that $\varphi_{\text {Ray }}$ will increase as $Q$ increases, favoring the semidilute entangled regime where $\varphi_{\text {Ray }}>\varphi_{\text {ent }}$ which generates fiber-free and spherical particles.

$$
\varphi_{\text {Ray }}=\varphi \frac{288 \varepsilon_{\text {air }} \varepsilon \gamma}{\left(\frac{I}{Q}\right)^{2} d^{3}}
$$

\subsubsection{Particle Size}

The microspheres produced for conditions 3 and 5-13 had narrow quasi-monodisperse size distributions with average diameters ranging from 10.64 to $17.80 \mu \mathrm{m}$ (standard deviations (SD) ranging from 2.05 to 4.93) (Figure 4). Electrospraying was shown to be reproducible for most conditions, with average particle sizes always comprised within a $3 \mu \mathrm{m}$ difference. A short tip-to-collector (TTC) distance, however, did not ensure reproducibility with $15 \mathrm{~cm}$ being too close (condition 4). Distances of $20 \mathrm{~cm}$ and $25 \mathrm{~cm}$ led to better reproducibility, with a trend showing a slightly narrower size distribution when using a slower feed rate $(0.2 \mathrm{~mL} / \mathrm{h}$ instead of $0.5 \mathrm{~mL} / \mathrm{h})$, as shown by standard deviations from Figure 4(d).

In accordance with previous studies, the flow rate and polymer concentration were the main parameters to tune particle size, while gauge had non-significant effect on particle size as shown by M7 and M8 sizes in Figure $4(21 \mathrm{G}$ versus $26 \mathrm{G})$. However, it was observed that the size distribution was slightly broader for the bigger gauge with $\mathrm{SD}=2.40$ for $26 \mathrm{G}(\mathrm{M} 7)$ and $\mathrm{SD}=3.42$ for the $21 \mathrm{G}$ (M8) (i.d. $=0.241 \mathrm{~mm}$ and $0.495 \mathrm{~mm}$, respectively). It is inferred from this result that a smaller gauge can produce a narrower size distribution. 
Figure 4. (a) Table of parameters for each electrospraying condition. (b) Average particle size of each replicate obtained per electrospraying condition (3 replicates per condition) expressed as box plots $(\mathrm{n}=100-1,000)$. (c) Histograms of size distributions. (d) Average particle size of each condition expressed as means and standard deviations. (e) Inset showing the average particle size (means \pm standard errors) as a function of the voltage, for 2 different rates $(0.2$ and $0.5 \mathrm{~mL} / \mathrm{h})$.

\begin{tabular}{|c|c|c|c|c|c|c|c|c|c|c|c|}
\hline Concentration $(\% \mathrm{w} / \mathrm{v})$ & 9 & 10 & 10 & 10 & 10 & 10 & 10 & 10 & 10 & 10 & 10 \\
\hline Voltage $(\mathrm{kV})$ & 10 & 10 & 10 & 10 & 10 & 10 & 10 & 16 & 16 & 18 & 18 \\
\hline TTC distance $(\mathrm{cm})$ & 25 & 15 & 20 & 20 & 25 & 25 & 25 & 25 & 25 & 25 & 25 \\
\hline Feed rate $(\mathrm{mL} / \mathrm{h})$ & 0.5 & 0.5 & 0.5 & 0.2 & 0.5 & 0.5 & 0.2 & 0.5 & 0.2 & 0.5 & 0.2 \\
\hline Gauge & 26 & 26 & 26 & 26 & 26 & 21 & 26 & 26 & 26 & 26 & 26 \\
\hline
\end{tabular}

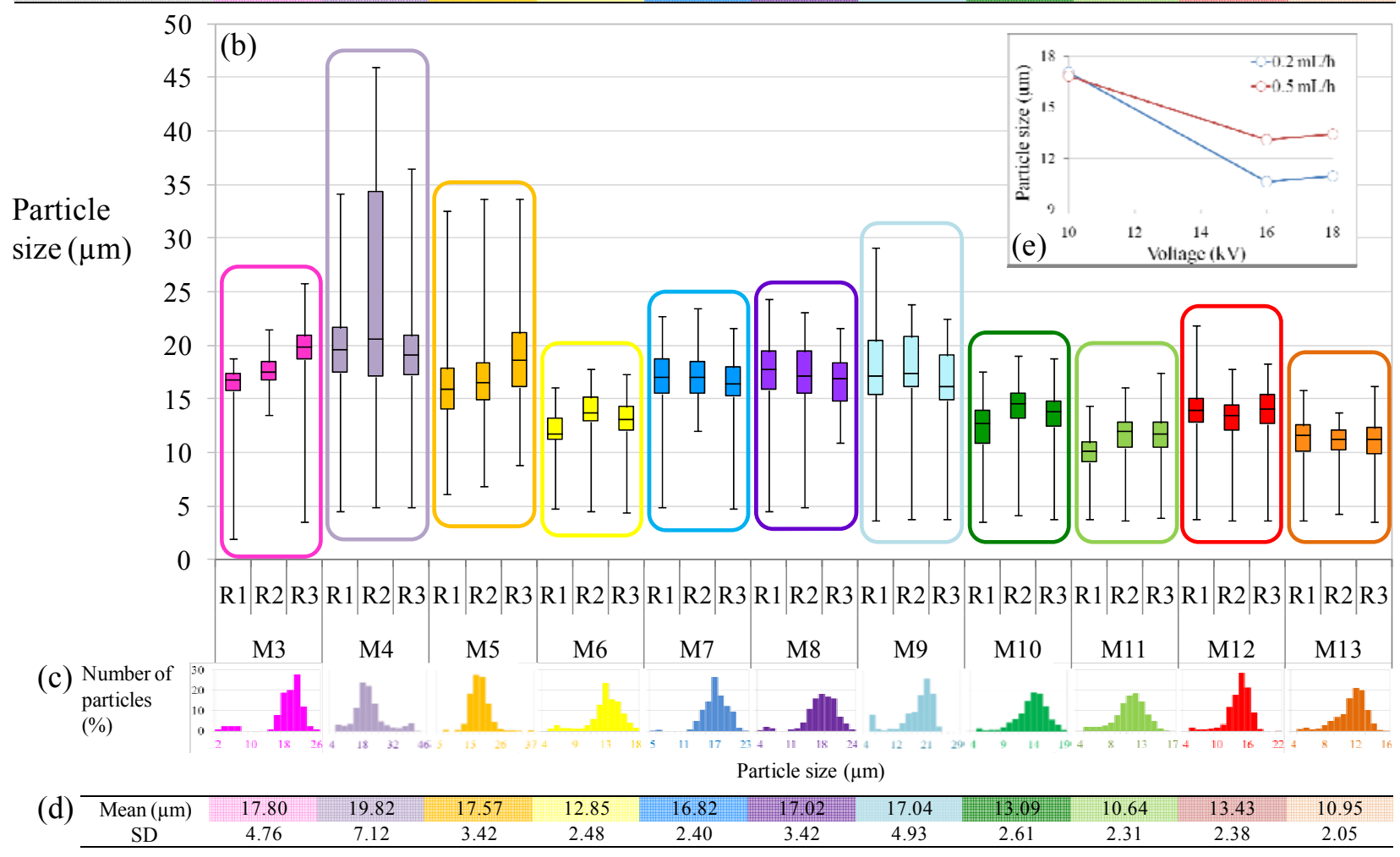

The polymer concentration impacts the surface tension of the solution, affecting particle size, as reported by Hartman et al. in the micro-dripping mode of electrospraying [39] and shown in Equations (4) and (5):

$$
\begin{aligned}
& d=\alpha\left(\frac{\rho \varepsilon_{0} Q^{4}}{I^{2}}\right)^{\frac{1}{6}} \\
& I \propto(\gamma K Q)^{\frac{1}{2}}
\end{aligned}
$$

where $d$ is the droplet diameter $(\mathrm{m}), \alpha$ is a constant, $\mathrm{Q}$ is the liquid flow rate $\left(\mathrm{m}^{3} . \mathrm{s}^{-1}\right), \rho$ is the solution density, $I$ is the current, $\varepsilon_{0}$ is the permittivity of vacuum, $\gamma$ is the surface tension of solution in ambient air, and $K$ is the liquid conductivity. These equations indicate that particle size increases with decreasing surface tension, thus implying decreased polymer content, and also increases for increasing flow rate. This is in accordance with the results of PCL microspheres where for similar spraying conditions (M3 and M7), the $9 \% \mathrm{w} / \mathrm{v}$ solution led to an average diameter of $17.8 \mu \mathrm{m}(\mathrm{SD}=4.76)$ 
versus $16.8 \mu \mathrm{m}(\mathrm{SD}=2.40)$ for the $10 \% \mathrm{w} / \mathrm{v}$ solution. Although slight, this difference is statistically significant with $\mathrm{p}<0.05$ (t-test assuming equal variances, $\mathrm{n}>100$ ) and is due to the closeness of the solution concentrations ( 9 and $10 \% \mathrm{w} / \mathrm{v})$. However, for any drug release application, it must be noted that such a small size difference would likely not lead to dramatic differences in release profiles.

Interestingly, although voltage is known to have very little effect on particle size in PLA based polymers [36], we observed a significant effect from $10 \mathrm{kV}$ to voltages $\geq 16 \mathrm{kV}$ for PCL microspheres. At 16 and $18 \mathrm{kV}$, no significant differences in particle size were observed for each feed rate, as shown by the inset in Figure 5, and as expected from the theory, particle size was only decreased at low feed rates $(0.2 \mathrm{~mL} / \mathrm{h}$ versus $0.5 \mathrm{~mL} / \mathrm{h})$. However, at $10 \mathrm{kV}$, particle size was significantly larger than at higher voltages, regardless of feed rates. It might be inferred that voltage has an influence on particle size, as shown by Equation (4) where particle size decreases with increasing current. The reason for this decrease is the presence of fibers as confirmed by the morphology images (Figure 3 ). In fact, in Figures 3(a,b) and 3(c,d), there is no significant increase in fiber formation for a decreased feed rate at a fixed voltage of $10 \mathrm{kV}$, and so there is no significant difference in particle size for that condition. However at 16 and $18 \mathrm{kV}$ the number of fibers is increased when decreasing the feed rate and the particle size is decreased accordingly. Therefore it can be concluded that this decrease in particle size is not only due to the increased voltage, but it is due to fiber formation occurring for a combination of lower feed rates and higher voltages. This can be confirmed with Equation (3), where $\varphi_{\text {Ray }}$ is decreased for increased voltages and decreased feed rates, falling in the case where $\varphi_{\mathrm{ov}}<\varphi_{\text {Ray }}<\varphi_{\text {ent }}$ and extruded particles are formed, instead of the ideal case where $\varphi_{\text {Ray }}>\varphi_{\text {ent }}$. As a consequence, the size of particles was reduced since a fraction of smaller offspring droplets were ejected from the initial droplet, drawing extruded fibers along. Using higher voltages are therefore to be used with care, and a sufficient high feed rate should be chosen to compensate the high voltage, for remaining in the regime where $\varphi_{\text {Ray }}>\varphi_{\text {ent }}$.

From the size distribution plots shown in Figure 4(c), a bimodal character could be observed for some conditions, made up of a majority of primary droplets and a small percentage of smaller particles (less than 5\%). These smaller droplets are offspring droplets caused by Coulomb fission [36,39]. They are easily ejected from the primary droplet during shrinkage of droplets occurring during evaporation. The offspring phenomenon was emphasized for decreased polymer concentration contents as shown by M3-type microspheres compared to M7-type microspheres ( 9 and $10 \% \mathrm{w} / \mathrm{v}$ respectively). This is likely due to decreased entanglement for decreased polymer contents, which favors the occurrence of offspring droplets. However, in this context, the frequency of offspring droplets is extremely low for most spraying conditions and thus would have no significant effect on release profiles in the case of drug loading.

\subsubsection{Reproducibility of Electrospraying}

To assess reproducibility of particle formation, condition 7 was used, 6 weeks apart. During this six week break the electrospraying setup was used by other researchers such that all the parameters had to be reset. The average particle size for the three runs at week zero was $16.82 \mu \mathrm{m}(\mathrm{n}=330, \mathrm{SD}=2.40)$ (Figure 4) while the repeat at 6 weeks had average particle size of $16.16 \mu \mathrm{m}(\mathrm{n}=289, \mathrm{SD}=3.98)$ with 
unchanged morphology. Apart from non-significant increase in size distribution, the two conditions were identified as identical.

The same reproducibility was not, however, observed for all conditions. For instance, conditions where flat particles were observed (conditions 1 and 2) intrinsically lacked reproducibility based on same-day repeats. These conditions reflected the semidilute unentangled regime where smaller offspring droplets were ejected and droplets were not fully dried.

For semidilute entangled regimes, shown for concentrations $>9 \% \mathrm{w} / \mathrm{v}$, more parameters will influence the reproducibility of electrospraying. For instance, a high TTC distance has to be ensured for complete solvent evaporation. If the TTC distance is too short, as in condition 4 (TTC $=15 \mathrm{~cm})$, entangled but not fully dry particles are produced, leading to very broad size distributions as seen in Figure 4(c). In this study, TTC distances of 20 and $25 \mathrm{~cm}$ were shown to be ideal in terms of reproducibility. The feed rates used were shown to generate reproducible samples either at $0.2 \mathrm{~mL} / \mathrm{h}$ or $0.5 \mathrm{~mL} / \mathrm{h}$, with the formation of fibers observed for the lowest feed rate, when working at high polymer concentration (conditions 9, 11 and 13). Although not ideal in terms of final morphology of microspheres, these conditions were shown to be reproducible from one replicate to the next.

An interesting observation was that although the change in voltage did not affect reproducibility in particle size and distribution we did notice several collection points of particles at high voltage, variable for each run, whereas at low voltage the pattern reflected one collection point, circular and centered. This may be attributed to the stable micro-dripping mode at low voltage versus the multi-dripping mode at high voltage as observed previously $[15,40]$. This may be an issue when the collector is a secondary scaffold, for example when making composite scaffolds.

To conclude, it must be understood that reproducibility can be achieved with electrospraying, but only for certain parameters intrinsic to each polymer solution. These parameters have to be determined and optimized first, so that the reproducibility of the process is ensured. The entanglement regime is the most important to start with and $c>c_{\text {ent }}$ has to be ensured to meet reproducibility while sufficiently high TTC distance is also important, to ensure full solvent evaporation.

\subsubsection{Thermal Characterization}

Differential scanning calorimetry (DSC) was used to ensure that chloroform and electrospraying process would not lower the crystallization of PCL, impacting on the characteristics of electrosprayed particles. The initial DSC run was followed by a repeat run with the thermal history erased in order to check the polymer itself. However, the study of the first run of each sample allowed assessing any eventual polymer discrepancies caused by the electrospraying process or contact with chloroform.

DSC results, presented in Figure 5, show single melting peaks with a maximum melting temperature $\left(\mathrm{T}_{\mathrm{m}}\right)$ of unprocessed PCL of $61.2{ }^{\circ} \mathrm{C}$ and a heat of melting $\left(\mathrm{H}_{\mathrm{m}}\right)$ of $63.6 \mathrm{~J} / \mathrm{g}$, while $\mathrm{T}_{\mathrm{m}}=58.9{ }^{\circ} \mathrm{C}$ and $\mathrm{H}_{\mathrm{m}}=71.9 \mathrm{~J} / \mathrm{g}$ for electrosprayed microspheres, translating respectively to $45.6 \%$ and $51.5 \%$ crystallinity (based on $\mathrm{H}_{\mathrm{m}}$ for $100 \%$ crystalline PCL of $139.5 \mathrm{~J} / \mathrm{g}$ [41]). $\mathrm{T}_{\mathrm{m}}$ is shown to be slightly decreased, inferring that smaller crystallites are formed during electrospraying. The degree of crystallinity of PCL after the second run, with the thermal history removed was shown to be $35.0 \%$ and $38.3 \%$ for unprocessed PCL and electrosprayed beads, respectively. These non-significant values confirm that no intrinsic changes were made to the polymer that went into contact with chloroform, 
and further electrosprayed. The use of chloroform to dissolve PCL can therefore be validated as an appropriate solvent for electrospraying PCL solutions.

Figure 5. DSC traces of electrosprayed M3-type microspheres and unprocessed PCL pellet, first runs, exothermic is up.

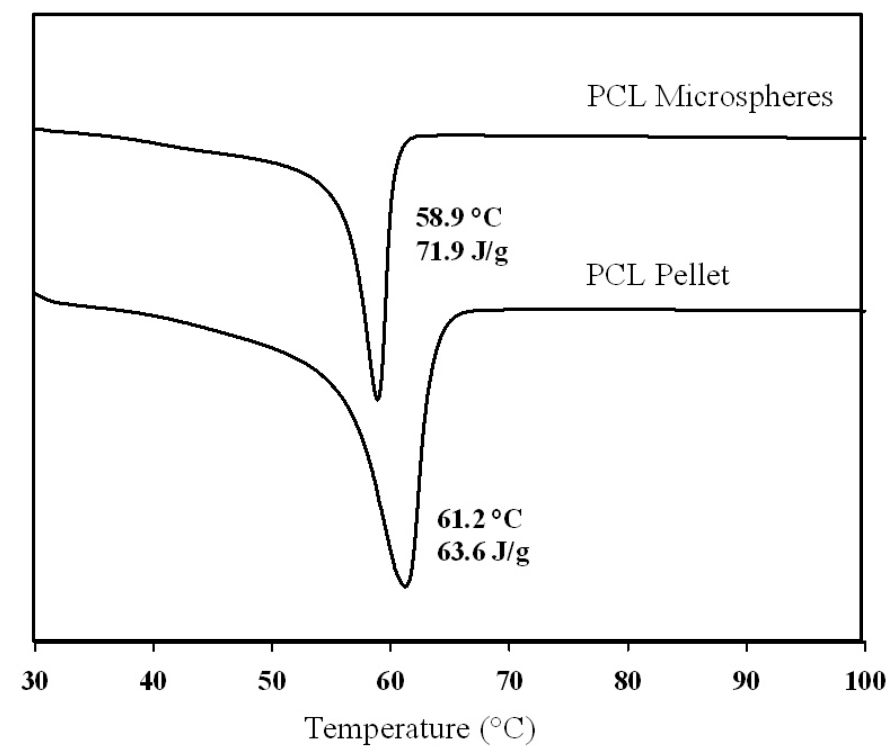

\subsection{Biological Effect of Microspheres}

Electrospraying remains a process that employs organic solvents. It is therefore important to ensure that these organic solvents are fully removed after process; otherwise the electrosprayed particles might be toxic to cells regardless of whether they comprise FDA-approved polymers. This is an important step which is often overlooked in many studies, where results are shown for loaded particles directly. However, when loading expensive growth factors, the risk of induced toxicity by other components is of concern. For this reason we tested the electrosprayed microspheres by a standard compatibility assay from the ISO 10993-12, called the elution or extract dilution assay, to ensure that no toxic residue is released. The microspheres were eluted into media for different times, which was then removed and seeded on a near-confluent monolayer of cells. If any chloroform residue, which is known to be highly toxic to cells, was extracted, the DNA quantification by the CyQUANT® assay was expected to be lower than the control. For the design of our experiment, we used 0.1 and $1 \% \mathrm{w} / \mathrm{v}$ of extract and incubated it for 1 and 24 hours, to probe for entrapped chloroform. The results are presented in Figure 6, which show no statistical difference between cells cultured with or without addition of extract solution in terms of DNA content, as stated by a two-way ANOVA analysis $(p>0.05)$. These preliminary results are a good indication that no chloroform is entrapped in electrosprayed beads, hence no adverse affect on the cells, and are in accordance with the DSC results above. However this study should be repeated for longer incubation times matching the whole course of PCL degradation. 
Figure 6. Results of CyQUANT® assay, expressing the DNA quantification after $48 \mathrm{~h}$ of exposure to cells (normalized to control), for different test solutions being incubated for different times before exposure to cells. $0.1 \% \mathrm{EX}$ and $1 \% \mathrm{EX}$ refer to 0.1 and $1 \% \mathrm{w} / \mathrm{v}$ extracts respectively, obtained from M3-type microspheres immersed in media, for different times. Results are expressed in normalized averages \pm standard errors $(n=4)$.

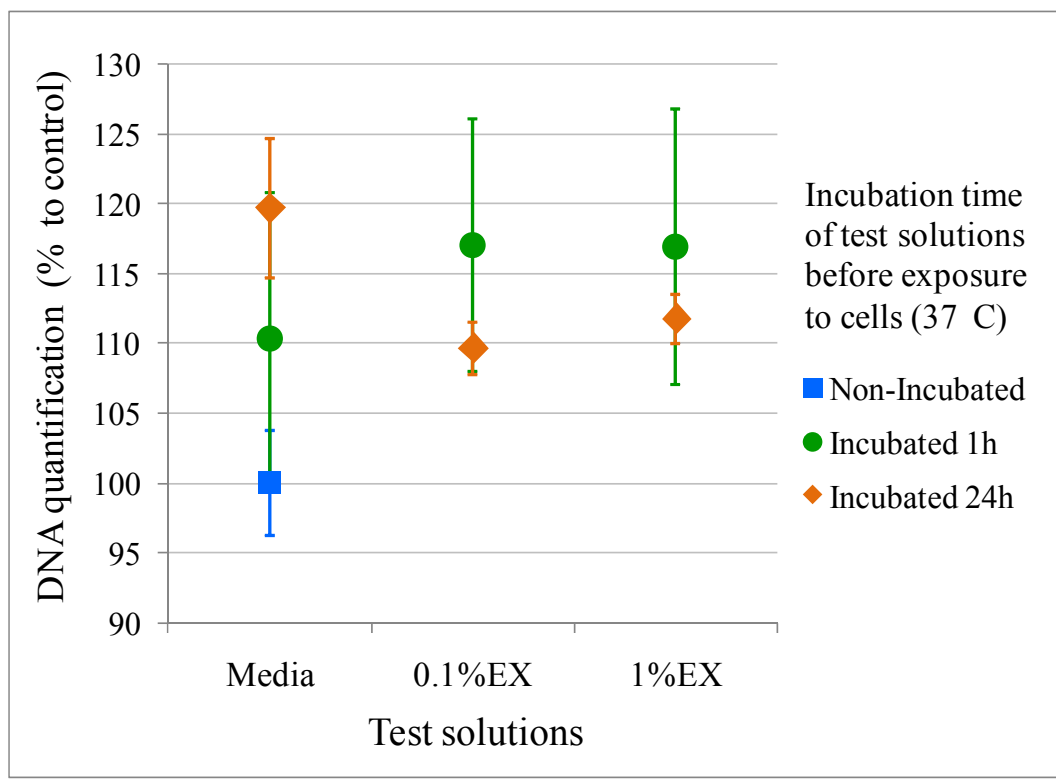

A direct contact assay from the same ISO 10993-12 was also performed to ensure that microspheres were physically compatible with cells. The microspheres were rinsed or left non-rinsed in media for different incubation times so that the effect of different conditions and times on cell viability could be assessed. Different concentrations of microspheres were used: $0.01 \%$ and $0.1 \% \mathrm{w} / \mathrm{v}$. It must be noted that such densities were extremely high relative to cell numbers and the study of cell morphology after contact with microspheres was not possible with microscopy since immersed microspheres would settle down on cells and completely cover them. However, after removal of microspheres, cell DNA contents were not lower than the controls for any of the condition tested and combinations (rinsed microspheres, non-rinsed microspheres, $1 \mathrm{~h}$ incubation, $24 \mathrm{~h}$ incubation), showing that microspheres did not have any physical adverse effects on cells (Figure 7), as determined by a two-way ANOVA analysis $(\mathrm{p}>0.05)$.

The CyQUANT® assay applied to toxicity determination only allows DNA quantification compared to a control. However in future studies, metabolic assays such as AlamarBlue or 3-(4,5-dimethylthiazol-2-yl)-2,5-diphenyl tetrazolium bromide (MTT) assays will give quantitative data regarding toxicity [42]. Subsequently, proliferative activity will be assessed with the CyQUANT ${ }^{\circledR}$ or PicoGreen assays ensuring that microspheres do not impair cell proliferation. 
Figure 7. Results of CyQUANT® assay, expressing the DNA quantification after $48 \mathrm{~h}$ of exposure to cells (normalized to control), for different test solutions being incubated for different times before exposure to cells. $0.01 \% \mathrm{NR}$ and $0.1 \% \mathrm{NR}$ refer to the 0.01 and $0.1 \% \mathrm{w} / \mathrm{v}$ M3-type microspheres rinsed in media before exposure. $0.01 \% \mathrm{R}$ and $0.1 \% \mathrm{R} \mathrm{w} / \mathrm{v}$ refer to the 0.01 and $0.1 \% \mathrm{w} / \mathrm{v}$ M3-type microspheres non-rinsed in media before exposure, but incubated for the same amount of time as rinsed microspheres. Results are expressed in normalized averages \pm standard errors $(n=4)$.

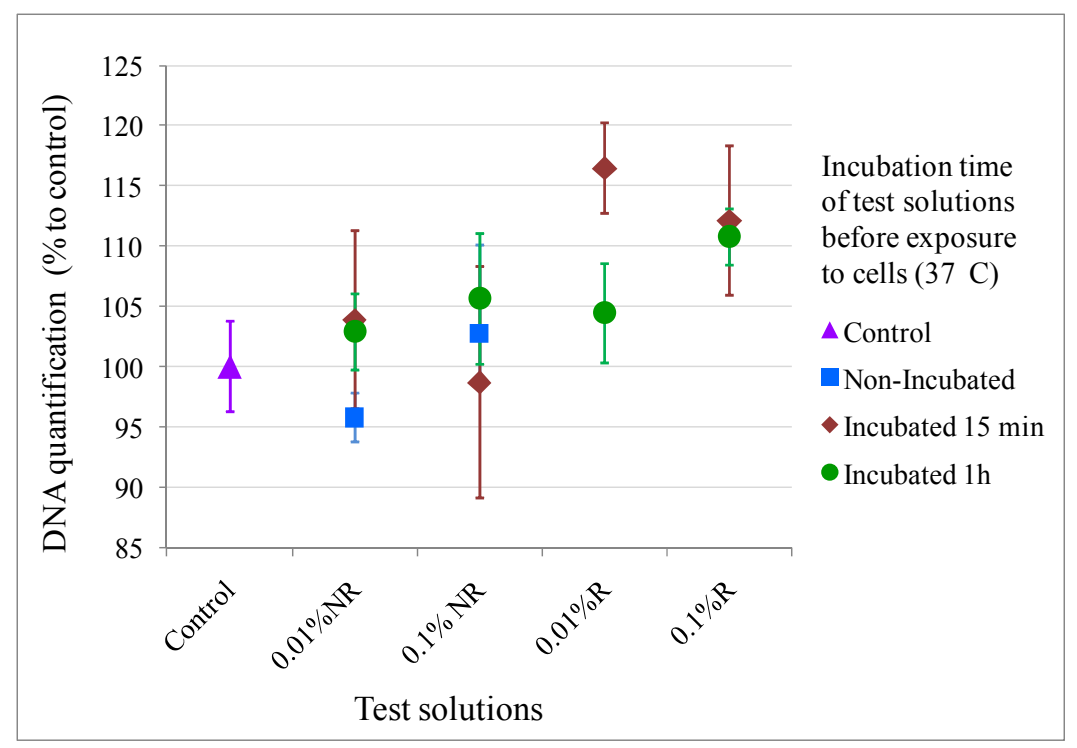

\section{Conclusions}

Electrospraying was shown to be a reproducible method for generating spherical PCL particles with narrow quasi-monodispere size distributions, with average sizes ranging from 10 to $20 \mu \mathrm{m}$, which could be tuned with electrospraying flow rate and polymer concentration. Control of particle morphology was shown to be tailored with these same variables by determining the polymer entanglement regime taking place in the course of electrospraying. In order to avoid fiber formation and offspring droplets, an increased polymer concentration must be coupled with an increased feed rate, ensuring electrospraying in the semidilute entangled regime, which leads to the formation of spherical, homogeneous and reproducible particles. Chloroform was shown to be an appropriate solvent for PCL particles, conferring a reproducible embossed texture to the electrosprayed microspheres, potentially beneficial for cell adherence. Chloroform did not act as a plasticizer in contact with PCL and was inferred to be fully removed after drying. Furthermore, electrosprayed microspheres showed no adverse effects on cell viability after $48 \mathrm{~h}$ exposure. In conclusion, this study has demonstrated precise control over polymer microsphere characteristics which may be used as a template for future microsphere-growth factor delivery systems.

\section{Acknowledgements}

T. R. D. acknowledges the Queensland Smart State Fellowship Scheme and Tissue Therapies Ltd. for financial support. M. A. W. is supported by the QUT Vice Chancellor's Fellowship Scheme and an ARC Linkage Grant (LP100200084). 


\section{References}

1. Freiberg, S.; Zhu, X. Polymer microspheres for controlled drug release. Int. J. Pharm. 2004, 282, $1-18$.

2. Park, J.S.; Lee, J.H.; Shin, H.S.; Lee, T.W.; Kim, M.S.; Khang, G.; Rhee, J.M.; Lee, H.K.; Lee, H.B. Biodegradable polymer microspheres for controlled drug release. Tissue Eng. Regenerative Med. 2007, 4, 347-359.

3. Ye, M.; Kim, S.; Park, K. Issues in long-term protein delivery using biodegradable microparticles. J. Control. Release 2010, 146, 241-260.

4. Sokolsky-Papkov, M.; Agashi, K.; Olaye, A.; Shakesheff, K.; Domb, A.J. Polymer carriers for drug delivery in tissue engineering. Adv. Drug Del. Rev. 2007, 59, 187-206.

5. Lassalle, V.; Ferreira, M.L. PLA nano- and microparticles for drug delivery: An overview of the methods of preparation. Macromol. Biosci. 2007, 7, 767-783.

6. Yang, Y.Y.; Chung, T.S.; Bai, X.L.; Chan, W.K. Effect of preparation conditions on morphology and release profiles of biodegradable polymeric microspheres containing protein fabricated by double-emulsion method. Chem. Eng. Sci. 2000, 55, 2223-2236.

7. Mundargi, R.C.; Babu, V.R.; Rangaswamy, V.; Patel, P.; Aminabhavi, T.M. Nano/micro technologies for delivering macromolecular therapeutics using poly(D,L-lactide-co-glycolide) and its derivatives. J. Control. Release 2008, 125, 193-209.

8. Kempen, D.H.R.; Kim, C.W.; Lu, L.; Dhert, W.J.A.; Currier, B.L.; Yaszemski, M.J. Controlled release from poly(lactic-co-glycolic acid) microspheres embedded in an injectable, biodegradable scaffold for bone tissue engineering. Mater. Sci. Forum 2003, doi: 10.4028/www.scientific.net/ MSF.426-432.3151.

9. Cohen, S.; Yoshioka, T.; Lucarelli, M.; Hwang, L.H.; Langer, R. Controlled delivery systems for proteins based on poly(lactic glycolic acid) microspheres Pharm. Res. 1991, 8, 713-720.

10. Carrascosa, C.; Torres-Aleman, I.; Lopez-Lopez, C.; Carro, E.; Espejo, L.; Torrado, S.; Torrado, J.J. Microspheres containing insulin-like growth factor I for treatment of chronic neurodegeneration. Biomaterials 2004, 25, 707-714.

11. Freitas, S.; Merkle, H.P.; Gander, B. Microencapsulation by solvent extraction/evaporation: Reviewing the state of the art of microsphere preparation process technology. J. Control. Release 2005, 102, 313-332.

12. Valo, H.; Peltonen, L.; Vehvilainen, S.; Karjalainen, M.; Kostiainen, R.; Laaksonen, T.; Hirvonen, J. Electrospray encapsulation of hydrophilic and hydrophobic drugs in poly(L-lactic acid) nanoparticles. Small 2009, 5, 1791-1798.

13. Ding, L.; Lee, T.; Wang, C.H. Fabrication of monodispersed Taxol-loaded particles using electrohydrodynamic atomization. J. Control. Release 2005, 102, 395-413.

14. Arya, N.; Chakraborty, S.; Dube, N.; Katti, D.S. Electrospraying: A facile technique for synthesis of chitosan-based micro/nanospheres for drug delivery applications. J. Biomed. Mater. Res. B-Appl. Biomater. 2009, 88B, 17-31.

15. Park, C.H.; Lee, J. Electrosprayed polymer particles: Effect of the solvent properties. J. Appl. Polym. Sci. 2009, 114, 430-437. 
16. Rayleigh, L. On the equilibrium of liquid conducting masses charged with electricity. Phil. Mag. Ser. 5 1882, 14, 184-186.

17. Zeleny, J. The electrical discharge from liquid points, and a hydrostatic method of measuring the electric intensity at their surfaces. Phys. Rev. 1914, 3, 69-91.

18. Taylor, G. Disintegration of water drops in an electric field. Proc. Roy. Soc. London Ser. A Math. Phys. Sci. 1964, 280, 383-397.

19. Shenoy, S.L.; Bates, W.D.; Frisch, H.L.; Wnek, G.E. Role of chain entanglements on fiber formation during electrospinning of polymer solutions: Good solvent, non-specific polymer-polymer interaction limit. Polymer 2005, 46, 3372-3384.

20. Gupta, P.; Elkins, C.; Long, T.E.; Wilkes, G.L. Electrospinning of linear homopolymers of poly(methyl methacrylate): Exploring relationships between fiber formation, viscosity, molecular weight and concentration in a good solvent. Polymer 2005, 46, 4799-4810.

21. Xu, Y.X.; Hanna, M.A. Electrospray encapsulation of water-soluble protein with polylactideEffects of formulations on morphology, encapsulation efficiency and release profile of particles. Int. J. Pharm. 2006, 320, 30-36.

22. Amsden, B.G.; Goosen, M.F.A. An examination of factors affecting the size, distribution and release characteristics of polymer microbeads made using electrostatics. J. Control. Release 1997, 43, 183-196.

23. Xu, Y.X.; Hanna, M.A. Electrosprayed bovine serum albumin-loaded tripolyphosphate cross-linked chitosan capsules: Synthesis and characterization. J. Microencapsul. 2007, 24, 143-151.

24. Xie, J.W.; Lim, L.K.; Phua, Y.Y.; Hua, J.S.; Wang, C.H. Electrohydrodynamic atomization for biodegradable polymeric particle production. J. Colloid Interface Sci. 2006, 302, 103-112.

25. Xu, Y.X.; Skotak, M.; Hanna, M. Electrospray encapsulation of water-soluble protein with polylactide. I. Effects of formulations and process on morphology and particle size. $J$. Microencapsul. 2006, 23, 69-78.

26. Hong, Y.L.; Li, Y.Y.; Yin, Y.Z.; Li, D.M.; Zou, G.T. Electrohydrodynamic atomization of quasi-monodisperse drug-loaded spherical/wrinkled microparticles. J. Aerosol Sci. 2008, 39, 525-536.

27. Hao, X.F.; Lu, X.F.; Li, Z.Y.; Zhao, Y.Y.; Shang, T.C.; Yang, Q.B.; Wang, C.; Li, L.J. Effects of the electrospray ionization parameters on the formation and morphology of colloidal microspheres of polyacrylonitrile. J. Appl. Polym. Sci. 2006, 102, 2889-2893.

28. Almeria, B.; Deng, W.W.; Fahmy, T.M.; Gomez, A. Controlling the morphology of electrospray-generated PLGA microparticles for drug delivery. J. Colloid Interface Sci. 2010, 343, 125-133.

29. Fantini, D.; Zanetti, M.; Costa, L. Polystyrene microspheres and nanospheres produced by electrospray. Macromol. Rapid Commun. 2006, 27, 2038-2042.

30. Loscertales, I.G.; Barrero, A.; Guerrero, I.; Cortijo, R.; Marquez, M.; Ganan-Calvo, A.M. Micro/nano encapsutation via electrified coaxial liquid jets. Science 2002, 295, 1695-1698.

31. Sinha, V.R.; Bansal, K.; Kaushik, R.; Kumria, R.; Trehan, A. Poly-epsilon-caprolactone microspheres and nanospheres: An overview. Int. J. Pharm. 2004, 278, 1-23. 
32. Woodruff, M.A.; Hutmacher, D.W. The return of a forgotten polymer-Polycaprolactone in the 21st century. Prog. Polym. Sci. 2010, 35, 1217-1256.

33. Wu, Y.Q.; Kennedy, S.J.; Clark, R.L. Polymeric particle formation through electrospraying at low atmospheric pressure. J. Biomed. Mater. Res. B-Appl. Biomater. 2009, 90B, 381-387.

34. Xie, J.W.; Wang, C.H. Encapsulation of proteins in biodegradable polymeric microparticles using electrospray in the Taylor Cone-Jet mode. Biotechnol. Bioeng. 2007, 97, 1278-1290.

35. Hwang, Y.K.; Jeong, U.; Cho, E.C. Production of uniform-sized polymer core-shell microcapsules by coaxial electrospraying. Langmuir 2008, 24, 2446-2451.

36. Meng, F.Z.; Jiang, Y.; Sun, Z.H.; Yin, Y.Z.; Li, Y.Y. Electrohydrodynamic liquid atomization of biodegradable polymer microparticles: Effect of electrohydrodynamic liquid atomization variables on microparticles. J. Appl. Polym. Sci. 2009, 113, 526-534.

37. Nie, H.; Dong, Z.; Arifin, D.Y.; Hu, Y.; Wang, C.-H. Core/shell microspheres via coaxial electrohydrodynamic atomization for sequential and parallel release of drugs. J. Biomed. Mater. Res. A 2010, 95A, 709-716.

38. Place, E.S.; Evans, N.D.; Stevens, M.M. Complexity in biomaterials for tissue engineering. Nat. Mater. 2009, 8, 457-470.

39. Hartman, R.P.A.; Brunner, D.J.; Camelot, D.M.A.; Marijnissen, J.C.M.; Scarlett, B. Jet break-up in electrohydrodynamic atomization in the cone-jet mode. J. Aerosol. Sci. 2000, 31, 65-95.

40. Jaworek, A.; Sobczyk, A.T. Electrospraying route to nanotechnology: An overview. J. Electrostat. 2008, 66, 197-219.

41. Crescenzi, V.; Manzini, G.; Calzolari, G.; Borri, C. Thermodynamics of fusion of poly- $\beta$-propiolactone and poly- $\varepsilon$-caprolactone. Comparative analysis of the melting of aliphatic polylactone and polyester chains. Eur. Polym. J. 1972, 8, 449-463.

42. Quent, V.M.C.; Loessner, D.; Friis, T.; Reichert, J.C.; Hutmacher, D.W. Discrepancies between metabolic activity and DNA content as tool to assess cell proliferation in cancer research. J. Cell. Mol. Med. 2010, 14, 1003-1013.

(C) 2011 by the authors; licensee MDPI, Basel, Switzerland. This article is an open access article distributed under the terms and conditions of the Creative Commons Attribution license (http://creativecommons.org/licenses/by/3.0/). 